\title{
1 Multi-sensor cloud and cloud shadow segmentation with a convolutional 2 neural network
}

3 Marc Wieland ${ }^{1}{ }^{*}, \mathrm{Yu} \mathrm{Li}{ }^{1}$, Sandro Martinis ${ }^{1}$

$4{ }^{1}$ German Remote Sensing Data Center (DFD), German Aerospace Center (DLR), Oberpfaffenhofen, D-82234

$5 \quad$ Wessling, Germany

6 * Correspondence: marc.wieland@dlr.de

7

8 Abstract

9 Cloud and cloud shadow segmentation is a crucial pre-processing step for any application that uses multi10 spectral satellite images. In particular, disaster related applications (e.g., flood monitoring or rapid damage 11 mapping), which are highly time- and data-critical, require methods that produce accurate cloud and cloud shadow masks in short time while being able to adapt to large variations in the target domain (induced by atmospheric conditions, different sensors, scene properties, etc.). In this study, we propose a data-driven approach to semantic segmentation of cloud and cloud shadow in single date images based on a modified U-Net convolutional neural network that aims to fulfil these requirements. We train the network on a global database of Landsat OLI images for the segmentation of five classes ("shadow", "cloud", "water", "land" and "snow/ice"). We compare the results to state-of-the-art methods, proof the model's generalization ability across multiple satellite sensors (Landsat TM, Landsat ETM+, Landsat OLI and Sentinel-2) and show the influence of different training strategies and spectral band combinations on the performance of the segmentation. Our method consistently outperforms Fmask and a traditional Random Forest classifier on a globally distributed multi-sensor test dataset in terms of accuracy, Cohen's Kappa coefficient, Dice coefficient and inference speed. The results indicate that a reduced feature space composed solely of red, green, blue and near-infrared bands already produces good results for all tested sensors. If available, adding shortwave-infrared bands can increase the accuracy. Contrast and brightness augmentations of the training data further improve the segmentation performance. The best performing U-Net model achieves an accuracy of 0.89, Kappa of 0.82 and Dice coefficient of 0.85 , while running the inference over 896 test image tiles with 44.8 seconds/megapixel (2.8 seconds/megapixel on GPU). The Random Forest classifier reaches an accuracy of 0.79, Kappa of 0.65 and Dice coefficient of 0.74 with 3.9 seconds/megapixel inference time (on CPU) on the same training and testing data. The rule-based Fmask method takes significantly longer (277.8 seconds/megapixel) and produces results with an accuracy of 0.75, Kappa of 0.60 and Dice coefficient of 0.72 .

\section{Keywords}




\section{Introduction}

34 Given current population growth rates and the increasingly visible effects of climate change on the environment and human activities, continuous monitoring of the spatio-temporal dynamics of earth surface processes is becoming ever more important. This becomes particularly prominent in emergency response applications for which near-real time geo-information about a disaster situation is crucial to target often limited resources and prioritize response actions (Voigt et al., 2016). To assure that earth observation based geo-information products have the highest possible spatial and temporal resolutions and information content, it is critical to simultaneously harvest data from a large variety of satellite sensors. While Synthetic Aperture Radar (SAR) sensors are widely used in emergency response applications due to their capability of penetrating through clouds and acquiring images during day and night, many tasks require the use of multi-spectral images to overcome limitations of SAR data and/or to improve the temporal resolution and timespan. In particular, time-series analysis benefit from the vast and consistent image archives of multi-spectral satellite sensors (Banskota et al., 2014). Also, urban (Wieland, 2016) and flood (Pekel et al., 2016) mapping and monitoring systems widely use multi-spectral images due to their extended thematic information content and simpler reflection characteristics in complex environments. The inherent potentials of multi-spectral satellite images are, however, being hampered by the presence of clouds and cloud shadows that may obstruct objects of interest and can bias image statistics, which can negatively impact on the performance of image analysis methods. Therefore, the usefulness of this imagery depends strongly on the ability to reliably mask clouds and cloud shadows from clear sky pixels. Being able to quickly and accurately identify cloud and cloud shadow pixels is fundamental for unbiased down-stream analysis and thus an essential step in producing analysis ready data.

Significant work has been undertaken to detect and segment clouds and cloud shadows in multi-spectral satellite images (Foga et al., 2017; Goodman and Henderson-Sellers, 1988; Pankiewicz, 2007). The existing methods can broadly be categorized into rule-based and machine learning approaches. The majority of rule-based methods exploit variations of reflectance in visible, shortwave-infrared and thermal bands and develop rule-sets that combine thresholds (Oishi et al., 2018) or functions over several spectral bands (Luo et al., 2008) to distinguish clouds from clear sky pixels. Cloud shadows are more difficult to detect than clouds, because their spectral signatures overlap with other dark surface materials (Main-Knorn et al., 2017). In particular, water surfaces are known to introduce false positives into shadow segmentation. Consequently, methods have been developed that consider spatial context information, sensor and solar geometry to estimate the occurrence of cloud shadows relative to the location of clouds (Hutchison et al., 2009). Zhu et al. (2012) (Zhu and Woodcock, 2012) introduce the Function of mask (Fmask) algorithm that uses rules based on physical cloud properties to identify potential cloud pixels from temperature, spectral variability, and brightness in Landsat TM and ETM+ images. The view angles of the satellite sensor, the illuminating angle and estimates of cloud altitude from thermal information are used to predict possible cloud shadow locations. Water and snow are reported as additional classes in their 
product. In a more recent study, they proposed an expansion of the Fmask algorithm to run on Landsat OLI and

Sentinel-2 (Zhu et al., 2015). Despite their wide usage, rule-based methods strongly depend on the particular sensor models, are sensitive to changes in atmospheric conditions and scene properties and lack generalization ability when transferring to other sensors. Multi-date methods use dense time-stacks of images acquired over the same location at different times to integrate temporal change vectors in their rule-sets for a more robust detection (Hagolle et al., 2010) (Roy et al., 2010). Their main limitation, however, is that they require a clear sky reference image and dense data, which increases operational costs and limits their applicability in time-critical emergency situations (Lyapustin et al., 2008).

In recent years, machine learning methods have been proposed to extract more robust high-level information from images (Bai et al., 2016; Lee and Lin, 2016). Li et al. (2017) (Li et al., 2017) use a Support Vector Machine to segment clouds from reflectance and texture information. Hollstein et al. (2016) (Hollstein et al., 2016) present an overview of several ready-to-use machine learning algorithms to detect "cloud", "cloud shadow", "snow/ice", "water", "flooded" and "clear sky" pixels in Sentinel-2 images. The algorithms they present include Classical Bayes, Decision Trees, Support Vector Machine and Stochastic Gradient Descent. Hughes and Hayes (2014) (Hughes and Hayes, 2014) introduce the Spatial Procedures for Automated Removal of Cloud and Shadow (SPARCS) algorithm that uses neural networks and rule-based post-processing to determine "cloud", "cloud shadow", "water", "snow/ice" and "clear sky pixels" in Landsat images. Convolutional Neural Networks (CNNs), which extract features directly from raw images by combining convolutional and pooling layers, gradually appear in recent studies on cloud segmentation (Chen et al., 2018; Mateo-Garcia et al., 2017; Zi et al., 2018). First results show superior accuracy, generalization ability and inference speed compared to rule-based and classical machine learning approaches. Zhaoxiang et al. (2018) (Zhaoxiang et al., 2018) use a lightweight UNet (Ronneberger et al., 2015) architecture and combine it with wavelet image compression for computationally efficient cloud detection on-board small satellites. Ozkan et al. (2018) (Ozkan et al., 2018) adapt a deep pyramid network to produce cloud masks from noisy labelled RGB colour images. Zhan et al. (2017) (Zhan et al., 2017) specifically focus on distinguishing cloud and snow from Gaofen1 imagery using a modified VGG network (Simonyan and Zisserman, 2014). Despite the promising results for cloud detection achieved in above mentioned deep learning studies, none of them specifically considers cloud shadows. Isikdogan, Bovik and Passalacqua (2017) (Isikdogan et al., 2017) use a Fully Convolutional Network for semantic segmentation of Landsat ETM+ images into five classes that include amongst others "cloud" and "shadow". Their main objective is large-scale surface water mapping, but generalization ability across sensors is not considered. Sholar (2018) (Sholar, 2017) confirms the observed lack of research and available deep learning models that specifically consider a shadow class and concludes that future work should focus on improving on this regard. Furthermore, additional research is needed to train models that generalize well across different sensors and images with varying atmospheric conditions and surface reflectance characteristics. 
$102 \mathrm{CNN}$ and trained on freely available reference data. We aim to fulfil the requirements imposed by emergency 103 response applications. In particular, our objective is to provide a rapid and simple to use method that is able to 104 mask cloud and cloud shadow pixels in single date images from a large variety of multi-spectral satellite sensors without the need for re-training or manual interference. We proof the generalization ability of our method across multiple satellite sensors (Landsat TM, Landsat ETM+, Landsat OLI and Sentinel-2) and compare it to the widely used Fmask algorithm (Zhu et al., 2015) and a traditional Random Forest classifier as baseline models. In this context, we also show the influence of different training strategies and spectral band combinations on the performance of the semantic segmentation. Compared to previous work in this direction, our study specifically focuses on multi-sensor generalization ability, simplicity and computational efficiency and provides an extensive performance evaluation across different sensors, seasons and locations. Furthermore, we consider a shadow class and distinguish between cloud, snow/ice, water and land pixels.

\section{Data}

114 In a supervised machine learning setup, a model is fit on a training dataset that is a set of labelled samples with 115 associated feature vector. For semantic segmentation tasks these are typically a labelled pixel mask and a multi116 band image. During hyperparameter tuning of the model, a validation dataset is used to provide an unbiased 117 evaluation of a model fit on the training dataset. The testing dataset is independent on the training and validation 118 data and provides an unbiased evaluation of a trained model. The freely available Spatial Procedures for 119 Automated Removal of Cloud and Shadow (SPARCS) dataset (USGS, 2016) is used in this study as training 120 dataset. The dataset was originally created and first described by Hughes and Hayes (2014) (Hughes and Hayes, 121 2014). It contains 80 subsets (1,000 x 1,000 pixels) of globally distributed Landsat OLI Level-1 scenes 122 accompanying manually delineated thematic masks (Figure 1). The masks contain the classes "cloud", "cloud 123 shadow", "snow/ice", "water", "flooded" and "land" and are provided in PNG image format. The Landsat 124 images are delivered with 11 spectral bands in GeoTIFF format with associated quality assessment band and metadata. In this paper, we use only spectral bands that are available across different satellite sensors to ensure a high degree of transferability of the trained models (Figure 2). Specifically, we use Landsat OLI bands 2 (Red),

1273 (Green), 4 (Blue), 5 (Near-Infrared NIR), 6 (Shortwave-Infrared SWIR1) and 7 (Shortwave-Infrared SWIR2).

128 We transform Digital Numbers (DN) to Top of Atmosphere (TOA) reflectance (USGS, 2013), convert the masks 129 into GeoTIFF format and combine classes "water" and "flooded", because the "flooded" class is underrepresented in the dataset and largely overlapping with the "water" class in terms of spectral response characteristics and thematic content. 


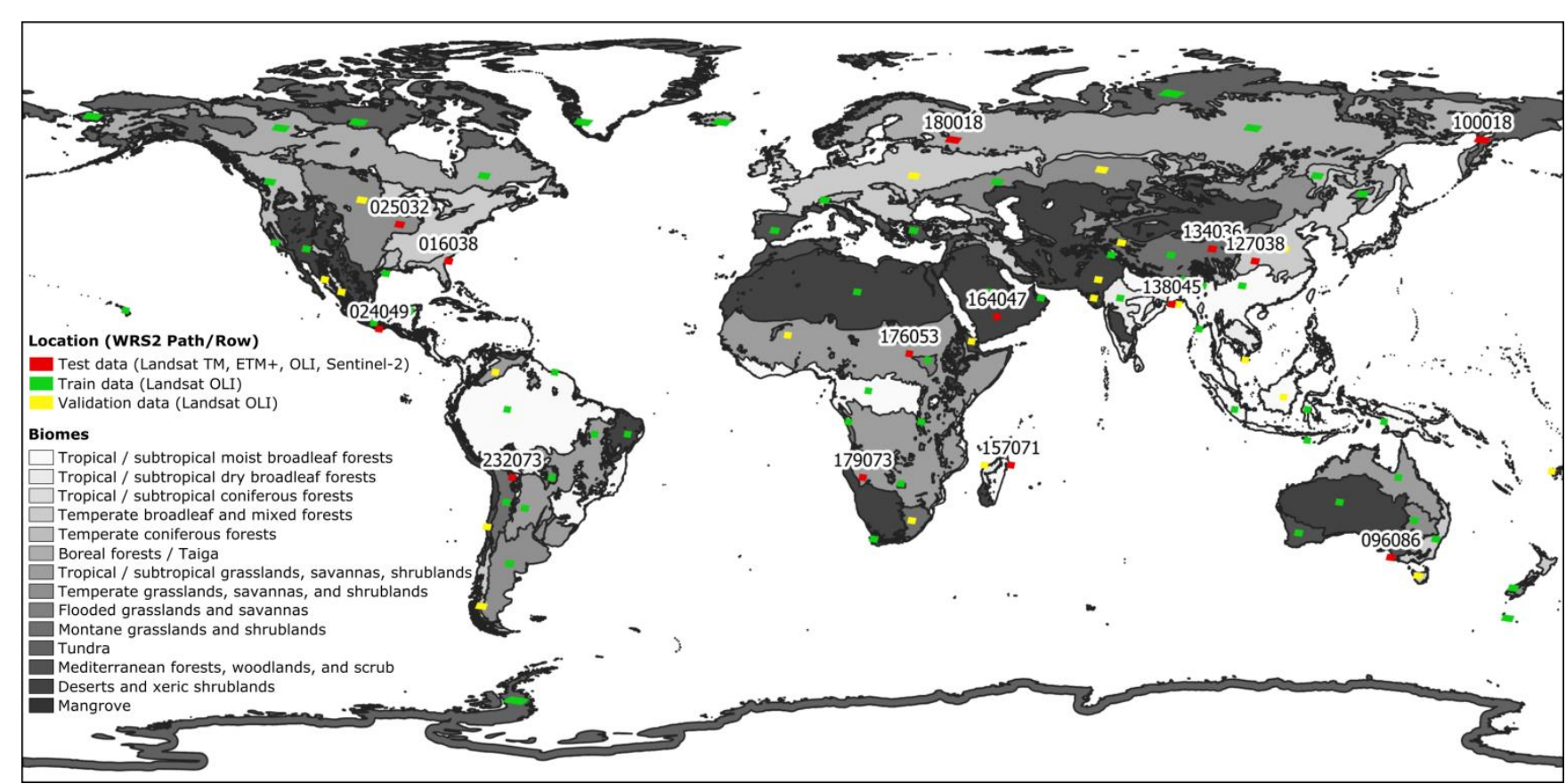

Figure 1: Worldwide Reference System 2 (WRS2) path/row locations of training, validation and testing data. Training and validation data are derived from the SPARCS dataset (Landsat OLI) (USGS, 2016). Stratified random sampling has been applied on the basis of a global biomes map (Olson et al., 2001) to choose WRS2 path/row locations for independent testing data from multiple sensors (Landsat TM, ETM+, OLI and Sentinel-2). Testing data samples are labelled with their unique identifier given by the respective path/row combination.

The processed images and accompanying masks are randomly split into training (70\%) and validation (30\%) datasets. In order to keep memory consumption low during training, we further split the training and validation datasets into tiles with 256 × 256 pixels size, which results in 896 tiles for training and 336 tiles for validation. Three augmentations with random contrast and brightness within +- $20 \%$ of the original values were created for each of the 896 training tiles, providing us with 3,584 tiles for training. Image augmentation allows the network to learn invariance to changes in the augmented domains to a degree that may go beyond what is present in the raw training images (Dosovitskiy et al., 2014). This is particularly important in remote sensing applications, since image properties are strongly affected by changes in atmospheric conditions, seasonality, land-use/landcover and other scene and image properties (sun elevation, radiometric resolution, etc.) and even a large training dataset may not cover all eventualities that may occur in real-world applications. Due to the spatial resolution of the input images and the natural variety of shapes of the classes of interest, we did not consider geometrical augmentations. In order to evaluate different training strategies on the performance of the segmentation, we define four training setups with varying number of spectral bands with and without augmentation. An overview of the training setups that we refer to throughout this study is provided in Table 1. 


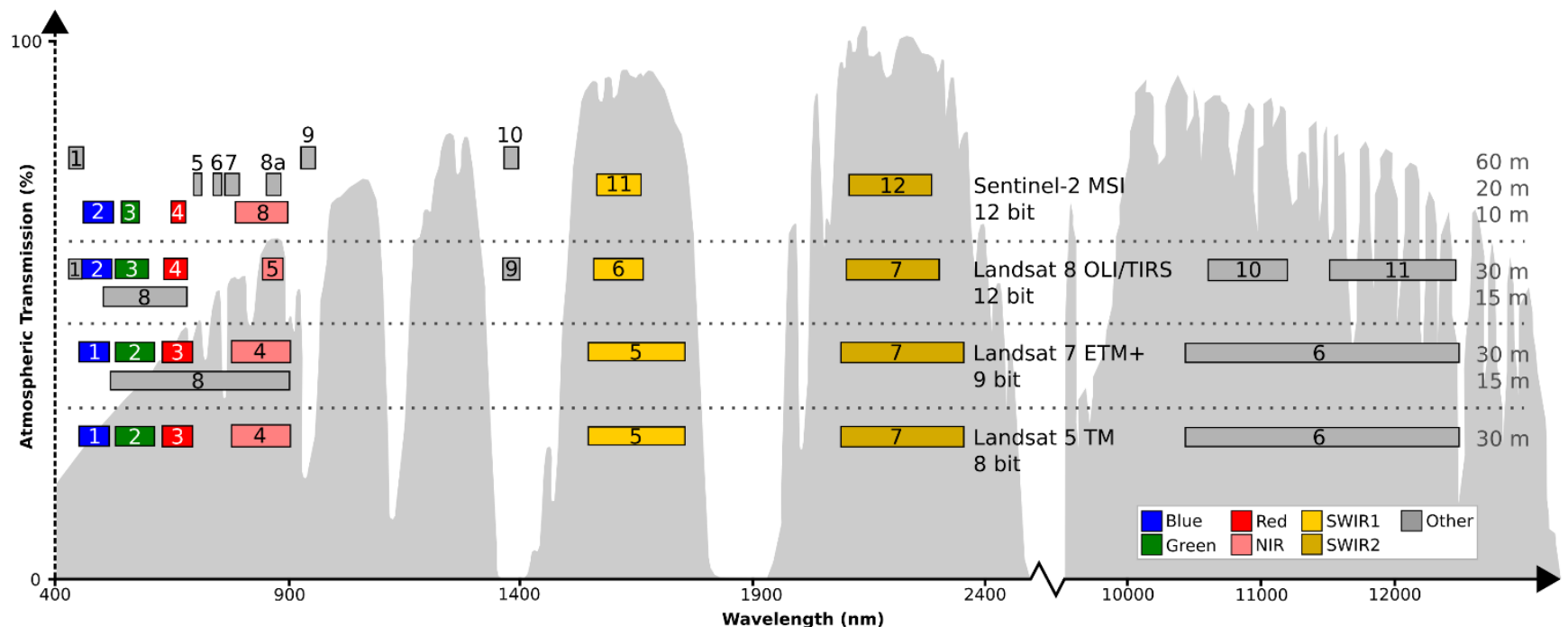

Figure 2: Comparison of spectral bands and spatial resolution across different satellite sensors superimposed on atmospheric transmission in grey. Modified after (USGS, 2018).

\begin{tabular}{|c|c|c|c|c|c|c|}
\hline ID & Sensor & Resolution & Spectral bands & Augmentation & Samples & Classes \\
\hline L8B6 & Landsat OLI & $30 \mathrm{~m}$ & Red, Green, Blue, NIR, SWIR1, SWIR2 & None & 896 & 5 \\
\hline L8B6A & Landsat OLI & $30 \mathrm{~m}$ & Red, Green, Blue, NIR, SWIR1, SWIR2 & Contrast/brightness & 3,584 & 5 \\
\hline L8B4 & Landsat OLI & $30 \mathrm{~m}$ & Red, Green, Blue, NIR & None & 896 & 5 \\
\hline L8B4A & Landsat OLI & $30 \mathrm{~m}$ & Red, Green, Blue, NIR & Contrast/brightness & 3,584 & 5 \\
\hline
\end{tabular}

Table 1: Training setups.

We take a stratified random sample of WRS2 path/row locations with a minimum distance constraint of $370 \mathrm{~km}$ (this equals twice the swath width of a Landsat scene) to identify 14 globally distributed locations, for which we acquire independent test images from Landsat TM, ETM+, OLI and Sentinel-2 sensors. The strata of our sampling scheme are formed by biomes that were derived from a global layer of terrestrial ecoregions (Olson et al., 2001). Biomes are distinct biological communities that have formed in response to shared environmental conditions. By stratifying over biomes we make sure that our test dataset covers a large variety of climatic, atmospheric and land-cover conditions. Figure 1 provides an overview of the Worldwide Reference System 2 (WRS2) path/row locations of imagery used in training, validation and testing. Acquisition times across test sample locations cover different seasons, whereas acquisition times across sensors at a single location are chosen as close as possible to each other to strengthen the significance of a cross-sensor comparison. The minimum cloud-cover percentage for image acquisitions was set to $5 \%$ in order to guarantee a certain degree of cloudcover per sample.

We resample Sentinel-2 data to $30 \mathrm{~m}$ to make sure that all images match the spatial resolution of the training and validation data. Furthermore, we create a 1,024 x 1,024 pixels subset for each test sample, stack the image bands together and convert DN to TOA reflectance. In case of Landsat images this is done by using radiometric rescaling coefficients provided in the metadata file of the Level-1 product. Sentinel-2 images are already 
delivered in TOA reflectance but need to be rescaled by a scaling factor that is provided in the respective metadata file. We also delineate scene classification masks that cover the same classes as our training dataset for each of the samples. Masks are generated manually by a single analyst by digitizing the respective images using QGIS software. Finally, we split the images and corresponding masks into 256 x 256 pixels tiles, which provides us with a total test data size of 896 tiles (224 tiles per sensor) for Landsat TM, ETM+, OLI and Sentinel-2.

\section{Method}

178 Figure 3 shows an overview of the performance evaluation framework that is used in this study to train, validate 179 and test different CNN models and compare them against Random Forest and Fmask benchmarking methods.

180

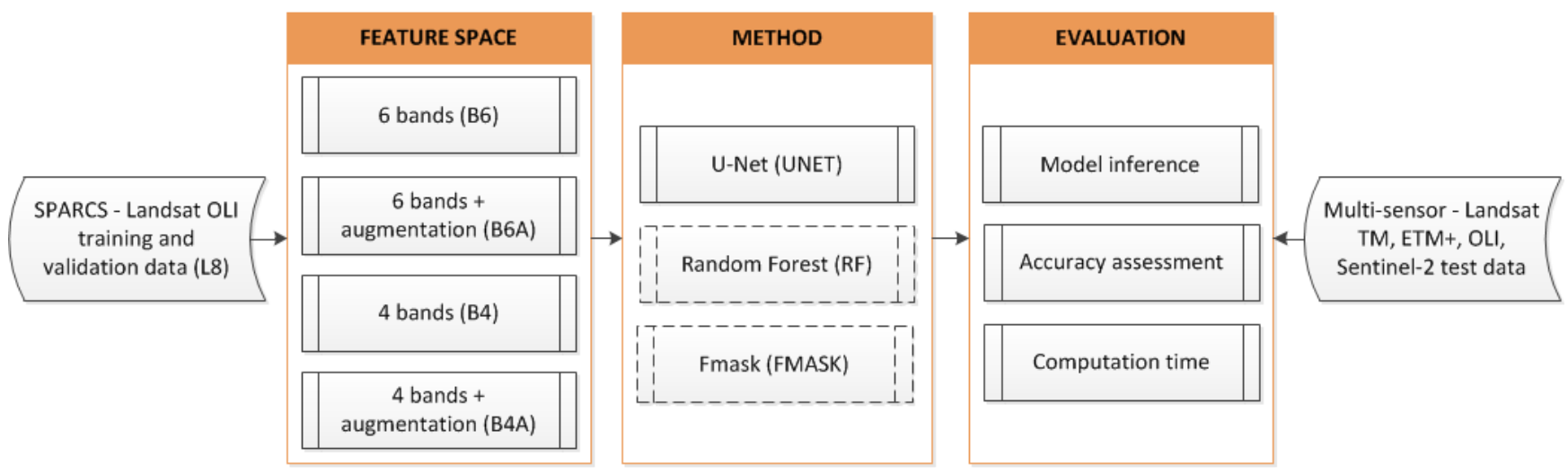

Figure 3: Overview of the performance evaluation framework.

Our CNN is based on the U-Net architecture (Ronneberger et al., 2015), which consists of encoder and decoder parts for semantic segmentation (Figure 4). The encoder takes an input image and generates a high-dimensional feature vector with aggregate features at multiple scales. In the elementary convolutional block we repeatedly apply two 3 x 3 convolutions with Rectified Linear Unit (ReLU) activation function, followed by batch normalization and a $2 \times 2$ max pooling operation with stride 1 for down-sampling. We use five convolutional blocks, where in each block the number of feature channels is doubled starting from 32. In the decoder part, the feature map is up-sampled by a 2 x 2 transpose convolution followed by a concatenation with the correspondingly cropped feature map from the decoder and two 3 × 3 convolutions with ReLU activation and batch normalization. This effectively halves the number of feature channels at each step. At the final layer a $1 \mathrm{x}$ 1 convolution with softmax activation function is used to map each feature vector $x$ to the desired number of classes $N($ Equation 1).

$$
\sigma(x)_{i}=\frac{e^{x_{i}}}{\sum_{n=1}^{N} e^{x_{n}}}
$$

The output of the network is a vector that contains the predicted class probabilities associated with the sample defined as in Equation 2 with $p_{i}(x)$ being the predicted probability of $x$ to belong to class $i$. 


$$
p(x)=\left\{p_{1}(x), p_{2}(x), \ldots, p_{i}(x), \ldots, p_{n}(x)\right\}
$$

195 The final categorical output $y$ of a given input is achieved by maximizing the corresponding predicted 196 probability vector $p(x)$ :

$$
y=\operatorname{argmax} p(x)
$$

197 The U-Net architecture was initially developed for bio-medical image segmentation. We chose U-Net as base 198 architecture, because of its capacity to learn from very little data while achieving state-of-the-art results on 199 semantic segmentation benchmark datasets (Ronneberger et al., 2015). It is, moreover, a lightweight network 200 with 7,766,981 trainable parameters for the given segmentation task and image type. Comparable state-of-the-art 201 architectures such as Fully Convolutional Networks are more complex with a higher amount of trainable 202 parameters and considerably longer training and inference times (Long et al., 2015). A common strategy to 203 reduce the computational burden during training of CNNs is to use a pre-trained base network and replace and 204 re-train the top layer of the classifier on a new dataset. Optionally also the weights of the pre-trained network 205 may be fine-tuned by backpropagation. In practise, this strategy works well when original and new datasets are 206 similar, such as natural pictures and very high resolution optical satellite images (Zhu et al., 2017). Given the difference between images that available pre-trained networks are trained on and medium resolution satellite images, which we target in this study, in terms of spectral bands and image scales, and given the availability of a large training dataset (with respect to the U-Net architecture), we decided to train the network from scratch. This, moreover, allowed for a greater flexibility in the network architecture.

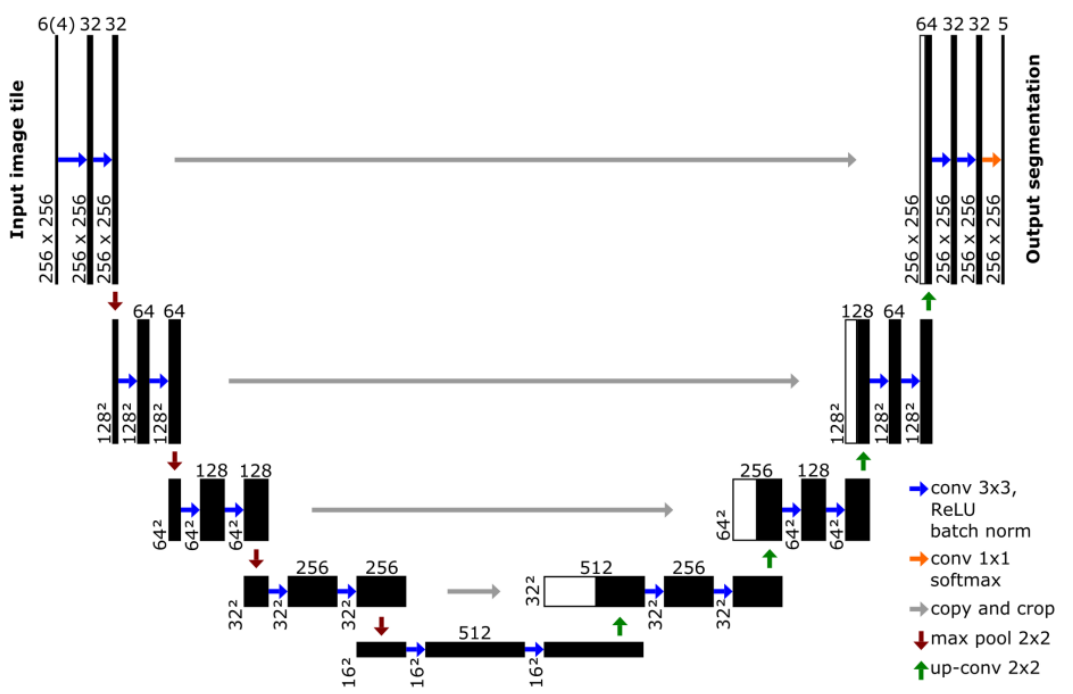

212 Figure 4: Architecture of the U-Net used in this study.

213 During training, we optimize the weights using adaptive moment estimation (Adam) algorithm (Kingma and Lei, 214 2015). We use default hyper-parameters $\beta_{1}=0.9$ and $\beta_{2}=0.999$ and set the initial learning rate based on the 215 method described in Smith (2017) (Smith, 2017). Even though Adam already computes adaptive learning rates 
216 to reduce the impact of tuning the hyper-parameters on convergence, it has been shown that additional learning 217 rate adaptation can improve convergence speed and accuracy (Smith, 2017). Therefore, we use a step decay 218 scheduler to further adapt the learning rate during training. We track several metrics for model evaluation, 219 including categorical cross-entropy loss, accuracy, Cohen's Kappa coefficient, Dice coefficient and inference 220 speed. Categorical cross-entropy loss is defined as in Equation 4 with $\hat{p}_{i}$ being the predicted probability for class $221 \quad i$ and $p_{i}$ being the true probability for that class.

$$
H(p, \hat{p})=-\sum_{i} p_{i} \log \left(\hat{p}_{i}\right)
$$

222 Accuracy is measured simply by the number of correctly classified pixels over the total number of pixels. A 223 more robust metric is Cohen's Kappa coefficient as it takes into account the possibility of the agreement 224 occurring by chance. It is defined as in Equation 5, where $p_{0}$ is the empirical probability of agreement on the 225 label assigned to any sample, and $p_{e}$ is the expected agreement when both classifiers assign labels randomly. $p_{e}$ 226 is estimated using a per-classifier empirical prior over the class labels.

$$
K=\frac{p_{0}-p_{e}}{1-p_{e}}
$$

227 In addition we also report the Dice coefficient, which is defined as twice the size of the intersection divided by 228 the sum of the size of each the predicted and the true categorical sample sets $A$ and $B$ (Equation 6).

$$
D(A, B)=\frac{2|A \cap B|}{|A|+|B|}
$$

Inference time (measured in seconds/megapixel) is included to account for the fact that our focus is on 230 applications in the emergency response sector, where computation time is a critical performance criterion. We 231 report CPU inference times, because only our U-Net implementation supports GPU inference, but not the 232 benchmark methods.

233 All experiments are carried out on a standard desktop PC with Intel Xeon CPU E5-1630 v4 @ 3.7 GHz, 4 cores 234 and 20 GB RAM. We use Keras with Tensorflow backend ("Keras," 2018) as deep learning framework and train 235 the network in batches of size 10 (to maximize GPU memory usage) over the four training datasets (Table 1) 236 separately until convergence (Figure 5). We standardize the input image feature space to zero mean and unit 237 variance with mean and standard deviation being computed on the training dataset and applied to the validation 238 and testing datasets, and shuffle the training set once between every training epoch. Training takes 239 approximately 1 hour for the datasets with augmentation and 20 minutes for the datasets without augmentation 240 on a NVIDIA M4000 GPU. 

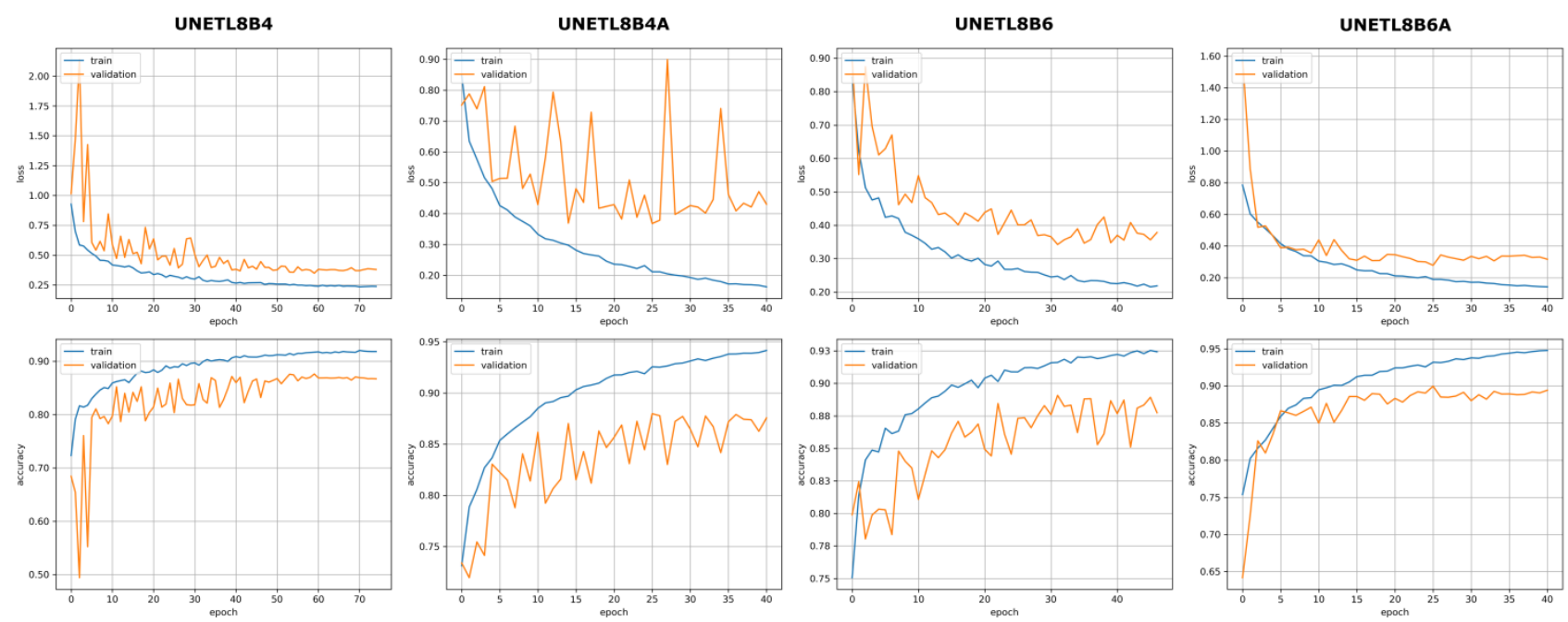

Figure 5: Visualization of the training history for U-Net models. A description of the different training setups can be found in Table 1 (e.g., UNETL8B4A refers to a U-Net model that has been trained on four Landsat OLI bands with contrast/brightness augmentation).

As a benchmark, we also train a traditional Random Forest classifier on the same standardized training datasets.

This classifier has been widely used in remote sensing applications and proved successful for the detection of clouds and cloud shadows in multi-spectral satellite images (Hollstein et al., 2016). We use the Scikit-learn ("Scikit-learn," 2018) implementation of the classifier for our experiments, which fits a number of optimized C4.5 decision tree classifiers (Breiman, 2001) on sub-samples of the training data and makes use of averaging to control over-fitting and improve the accuracy of the predictions. We tune hyper-parameters for each classification separately according to a ten-fold cross-validation and grid-search method during the training phase of the classifier.

Furthermore, we apply the rule-based Fmask algorithm (Zhu and Woodcock, 2012) to the test images and compare its results with our method. In contrast to the machine learning methods, the rule-sets that underlie Fmask are sensor specific and make use of all available spectral bands including thermal and cirrus bands where present (Zhu et al., 2015). In addition to that, the view angles of the satellite sensor, the illuminating angle and estimates of cloud altitude from thermal information are computed as intermediate products to feed into the prediction of possible cloud shadow locations. We use the pythonfmask package in version 0.5.2 in this study, which implements the Fmask algorithm as a Python module ("Python Fmask," 2018). According to the developers, with default settings the package should produce results that are equivalent to the masks that are included in the quality assessment layers of Landsat Collection-1 data.

\section{Results}

263 A description of the different training setups used for U-Net (UNET) and Random Forest (RF) classifiers can be found in Table 1 (e.g., UNETL8B4A refers to a U-Net model that has been trained on four Landsat OLI bands with contrast/brightness augmentation). Figure 6 and Table 2 show a summary of the results for each tested 
model grouped by sensor and considering all classes ("cloud", "cloud shadow", "snow/ice", "water" and "land"). 267 Over the globally distributed 896 test tiles for Landsat TM, ETM+, OLI and Sentinel-2, it can be seen that 268 UNETL8B6A (U-Net trained on six Landsat OLI bands with contrast/brightness augmentation) outperforms the other U-Net models with respect to accuracy, Cohen's Kappa coefficient and Dice coefficient. Only on the Landsat ETM+ testing dataset augmentation seems to not significantly affect the performance of the prediction and the model without augmentation shows slightly better performance metrics. On all other datasets the model trained with augmentation shows superior results. The best results are achieved on the Landsat OLI testing dataset, which is not surprising since the model has been trained on data of the same sensor. The performance difference between sensors is minor, which indicates good generalization ability of this model across sensors.

With mean accuracy of 0.89 , mean Kappa coefficient of 0.82 and mean Dice coefficient of 0.85 measured across all sensors, the UNETL8B6A model performs consistently better than the benchmarking methods. Fmask produces acceptable results that for Landsat TM are comparable with the performance of the U-Net models, but it still lacks behind the UNETL8B6A model. On the other test data even U-Net models that were trained without augmentation and on only four spectral bands show higher performance values compared to Fmask, which makes use of all the available spectral bands and requires ancillary information such as view and illumination angles. In general, it can be observed that Fmask performs better on Landsat data than on Sentinel-2 data. Random Forest performs consistently worse than the U-Net models but comparable to Fmask. On Landsat TM Fmask is superior to Random Forest, whereas on Sentinel-2 Random Forest produces more accurate predictions. Similarly to the U-Net models, Random Forest performs better when more spectral bands are included in the feature space. Augmentation, however, seems to have no significant influence on the prediction performance of Random Forest. It scores in terms of the faster inference time, which is in the order of $1 / 10$ of the inference time of the U-Net models (running on CPU) and even 1/60 of the Fmask inference time. Running the U-Net models on GPU, however, can significantly improve their inference speed beyond Random Forest on CPU.
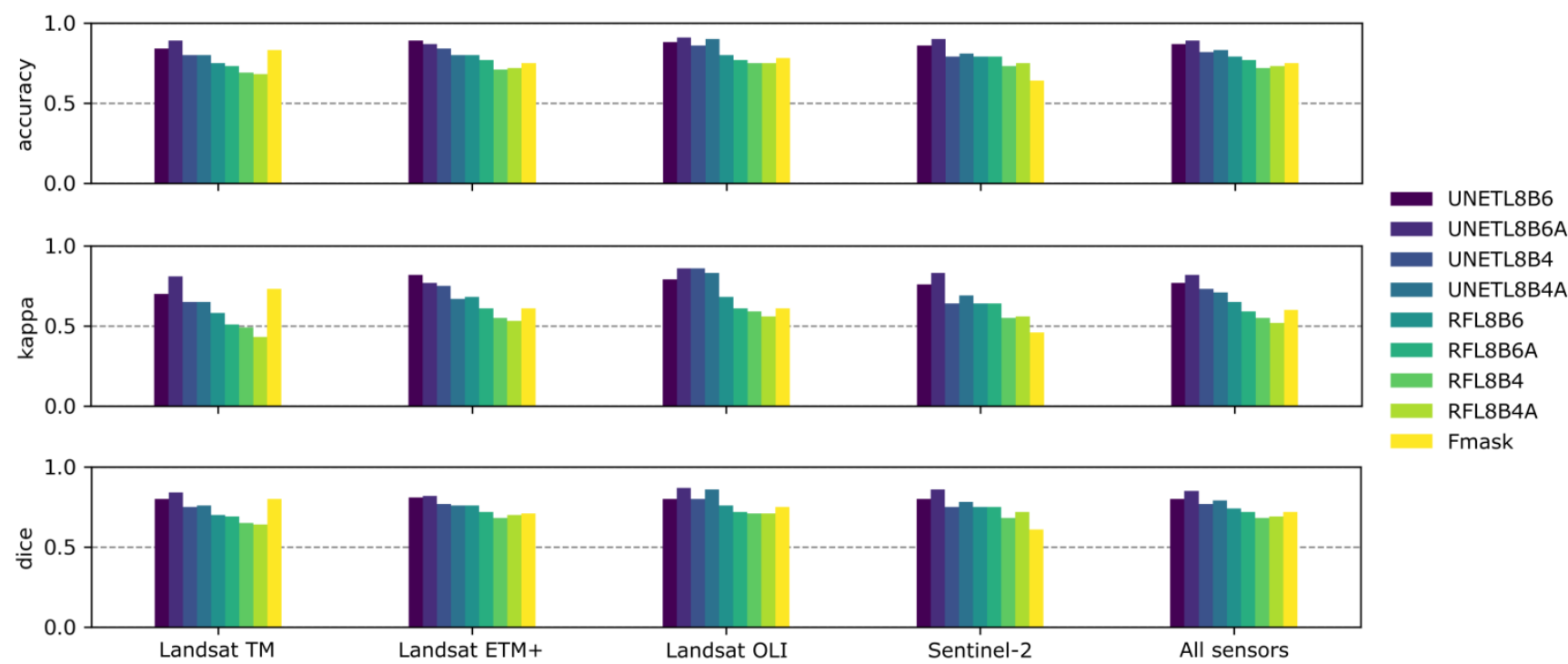
Fmask 


\begin{tabular}{|c|c|c|c|c|c|c|c|c|c|c|c|c|}
\hline \multirow{2}{*}{ Model } & \multicolumn{4}{|c|}{ Landsat TM } & \multicolumn{4}{|c|}{ Landsat ETM+ } & \multicolumn{4}{|c|}{ Landsat OLI } \\
\hline & Acc. & $\mathbf{K}$ & Dice & Time & Acc. & $\mathbf{K}$ & Dice & Time & Acc. & $\mathbf{K}$ & Dice & Time \\
\hline UNETL8B6 & 0.84 & 0.70 & 0.80 & $40.4(2.7)$ & 0.89 & 0.82 & 0.81 & $40.4(2.7)$ & 0.88 & 0.79 & 0.80 & $41.3(2.9)$ \\
\hline UNETL8B6A & 0.89 & 0.81 & 0.84 & $43.7(2.6)$ & 0.87 & 0.77 & 0.82 & $45.1(2.7)$ & 0.91 & 0.86 & 0.87 & $44.9(2.9)$ \\
\hline UNETL8B4 & 0.80 & 0.65 & 0.75 & $39.6(2.7)$ & 0.84 & 0.75 & 0.77 & $40.0(2.6)$ & 0.86 & 0.86 & 0.80 & $40.6(2.9)$ \\
\hline UNETL8B4A & 0.80 & 0.65 & 0.76 & $42.9(2.6)$ & 0.80 & 0.67 & 0.76 & $43.8(2.6)$ & 0.90 & 0.83 & 0.86 & $43.1(2.9)$ \\
\hline RFL8B6 & 0.75 & 0.58 & 0.70 & 4.1 & 0.80 & 0.68 & 0.76 & 4.0 & 0.80 & 0.68 & 0.76 & 4.1 \\
\hline RFL8B6A & 0.73 & 0.51 & 0.69 & 4.2 & 0.77 & 0.61 & 0.72 & 4.4 & 0.77 & 0.61 & 0.72 & 4.4 \\
\hline RFL8B4 & 0.69 & 0.49 & 0.65 & 4.0 & 0.71 & 0.55 & 0.68 & 3.9 & 0.75 & 0.59 & 0.71 & 3.9 \\
\hline RFL8B4A & 0.68 & 0.43 & 0.64 & 4.6 & 0.72 & 0.53 & 0.70 & 4.7 & 0.75 & 0.56 & 0.71 & 4.6 \\
\hline Fmask & 0.83 & 0.73 & 0.80 & 249.6 & 0.75 & 0.61 & 0.71 & 252.9 & 0.78 & 0.61 & 0.75 & 310.4 \\
\hline
\end{tabular}

Table 2 (Part1): Summary of the results for Landsat TM, ETM+, OLI and Sentinel-2 testing data. Times are in 292 seconds/megapixel for CPU inference on the test dataset. For U-Net additionally the GPU inference times are 293 provided in brackets.

\begin{tabular}{|l|c|c|c|c|c|c|c|c|}
\hline \multirow{2}{*}{ Model } & \multicolumn{3}{|c|}{ Sentinel-2 } & \multicolumn{3}{c|}{ All sensors $(\boldsymbol{\mu}+-\boldsymbol{\sigma})$} \\
\cline { 2 - 9 } & Acc. & $\mathbf{K}$ & Dice & Time & Acc. & K & Dice & Time \\
\hline UNETL8B6 & 0.86 & 0.76 & 0.80 & $41.9(2.9)$ & $0.87+-0.019$ & $0.77+-0.045$ & $0.80+-0.004$ & $41.0+-0.6(2.8+-0.1)$ \\
\hline UNETL8B6A & $\mathbf{0 . 9 0}$ & $\mathbf{0 . 8 3}$ & $\mathbf{0 . 8 6}$ & $45.4(3.1)$ & $\mathbf{0 . 8 9 + - 0 . 0 1 4}$ & $\mathbf{0 . 8 2 + - 0 . 0 3 3}$ & $\mathbf{0 . 8 5 + - 0 . 0 1 9}$ & $44.8+-0.6(2.8+-0.2)$ \\
\hline UNETL8B4 & 0.79 & 0.64 & 0.75 & $38.9(3.0)$ & $0.82+-0.029$ & $0.73+-0.089$ & $0.77+-0.020$ & $39.8+-0.6(2.8+-0.2)$ \\
\hline UNETL8B4A & 0.81 & 0.69 & 0.78 & $44.4(3.0)$ & $0.83+-0.042$ & $0.71+-0.071$ & $0.79+-0.041$ & $43.6+-0.6(2.8+-0.2)$ \\
\hline RFL8B6 & 0.79 & 0.64 & 0.75 & 4.0 & $0.79+-0.021$ & $0.65+-0.041$ & $0.74+-0.025$ & $4.1+-0.1$ \\
\hline RFL8B6A & 0.79 & 0.64 & 0.75 & 4.6 & $0.77+-0.022$ & $0.59+-0.049$ & $0.72+-0.021$ & $4.4+-0.1$ \\
\hline RFL8B4 & 0.73 & 0.55 & 0.68 & $\mathbf{3 . 9}$ & $0.72+-0.022$ & $0.55+-0.036$ & $0.68+-0.021$ & $\mathbf{3 . 9 + - 0 . 0 4}$ \\
\hline RFL8B4A & 0.75 & 0.56 & 0.72 & 4.6 & $0.73+-0.029$ & $0.52+-0.053$ & $0.69+-0.031$ & $4.6+-0.04$ \\
\hline Fmask & 0.64 & 0.46 & 0.61 & 298.3 & $0.75+-0.069$ & $0.60+-0.096$ & $0.72+-0.069$ & $277.8+-26.9$ \\
\hline
\end{tabular}

294 Table 2 (Part2): Summary of the results for Landsat TM, ETM+, OLI and Sentinel-2 testing data. Times are in 295 seconds/megapixel for CPU inference on the test dataset. For U-Net additionally the GPU inference times are 296 provided in brackets.

297 Figure 7 shows normalized error matrices for the best performing U-Net (UNETL8B6A) and Random Forest 298 (RFL8B6) models and Fmask over the combined test data for Landsat TM, ETM+, OLI and Sentinel-2. It can be 299 seen that all three models perform well for classes "land" and "cloud" with main differences coming from the U300 Net model being more precise (Table 3). Major misclassifications arise for the classes "shadow", "water" and 301 "land". The Random Forest RFL8B6 model falsely classifies "shadow" as "water" or "land", and "water" as 302 "shadow". The UNETL8B6A model significantly improves precision and recall for these classes, but still shows 303 a tendency to misclassify "shadow", "water" and "land". The model also reduces the confusion between 304 "snow/ice" and "cloud" as well as "land" and "cloud" compared to the other methods. Fmask shows high recall 
for the "cloud" class but the results indicate problems related to distinguishing "snow/ice" from "cloud". Moreover, a significant amount of "shadow" is falsely classified as belonging to the "cloud" and "land" classes. Confusion between "water" and "shadow" is present in the Fmask results, but it is not as strong as for Random 8).
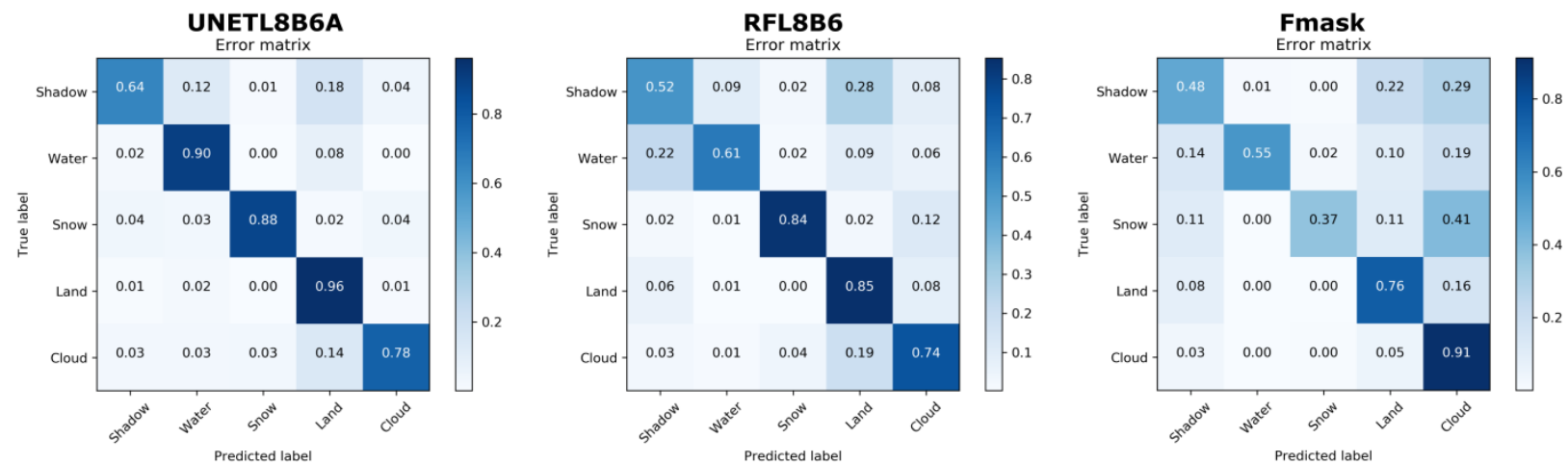

310

Figure 7: Error matrices for the best performing U-Net and Random Forest models and Fmask over the 896 test tiles for Landsat TM, ETM+, OLI and Sentinel-2.

\begin{tabular}{|c|c|c|c|c|c|c|c|c|c|}
\hline \multirow{2}{*}{ Class } & \multicolumn{3}{|c|}{ UNETL8B6A } & \multicolumn{3}{c|}{ RFL8B6 } & \multicolumn{3}{c|}{ Fmask } \\
\cline { 2 - 11 } & Precision & Recall & F1-score & Precision & Recall & F1-score & Precision & Recall & F1-score \\
\hline Shadow & 0.73 & 0.64 & 0.69 & 0.34 & 0.52 & 0.41 & 0.31 & 0.48 & 0.38 \\
\hline Water & 0.77 & 0.90 & 0.83 & 0.83 & 0.61 & 0.70 & 0.96 & 0.55 & 0.70 \\
\hline Snow & 0.81 & 0.88 & 0.85 & 0.73 & 0.84 & 0.78 & 0.76 & 0.37 & 0.50 \\
\hline Land & 0.92 & 0.96 & 0.94 & 0.88 & 0.85 & 0.87 & 0.92 & 0.76 & 0.83 \\
\hline Cloud & 0.94 & 0.78 & 0.85 & 0.73 & 0.74 & 0.73 & 0.62 & 0.91 & 0.74 \\
\hline Total & 0.89 & 0.89 & 0.89 & 0.80 & 0.78 & 0.79 & 0.81 & 0.75 & 0.76 \\
\hline
\end{tabular}

Table 3: Per-class comparison between best performing U-Net and Random Forest models and Fmask over the 896 test tiles for Landsat TM, ETM+, OLI and Sentinel-2.

Figure 9 summarizes the results for the best performing U-Net (UNETL8B6A) and Random Forest (RFL8B6) models and Fmask over the combined test data for Landsat TM, ETM+, OLI and Sentinel-2 grouped by location (see Figure 1 for details about locations). The U-Net model shows the highest performance values at all locations except location 127038 (“Temperate broadleaf and mixed forests") where the Random Forest model performs marginally better in terms of Kappa coefficient. The range of Kappa values across locations is higher for Random Forest $(\Delta \mathrm{K}=0.50[0.26,0.76])$ and Fmask $(\Delta \mathrm{K}=0.47[0.29,0.76])$ than for U-Net, which shows more stable behaviour across locations with $\Delta \mathrm{K}=0.24[0.66,0.90]$. This indicates, moreover, that performance variations across locations are higher than the variations across sensors (RFL8B6 $\Delta K=0.10$ [0.58, 0.68], Fmask $\Delta \mathrm{K}=0.27[0.46,0.73]$ and UNETL8B6A $\Delta \mathrm{K}=0.09$ [0.77, 0.86]). Location 134036 ("Montane grasslands and shrublands") shows the lowest Kappa values, whereas location 157071 ("Tropical and subtropical moist 
broadleaf forests") is the best segmented by all models. The location with highest inter-model variation is 025032 ("Temperate grasslands, savannas, and shrublands"), and the location for which the different models

327 show the most comparable performance is 127038 ("Temperate broadleaf and mixed forests").
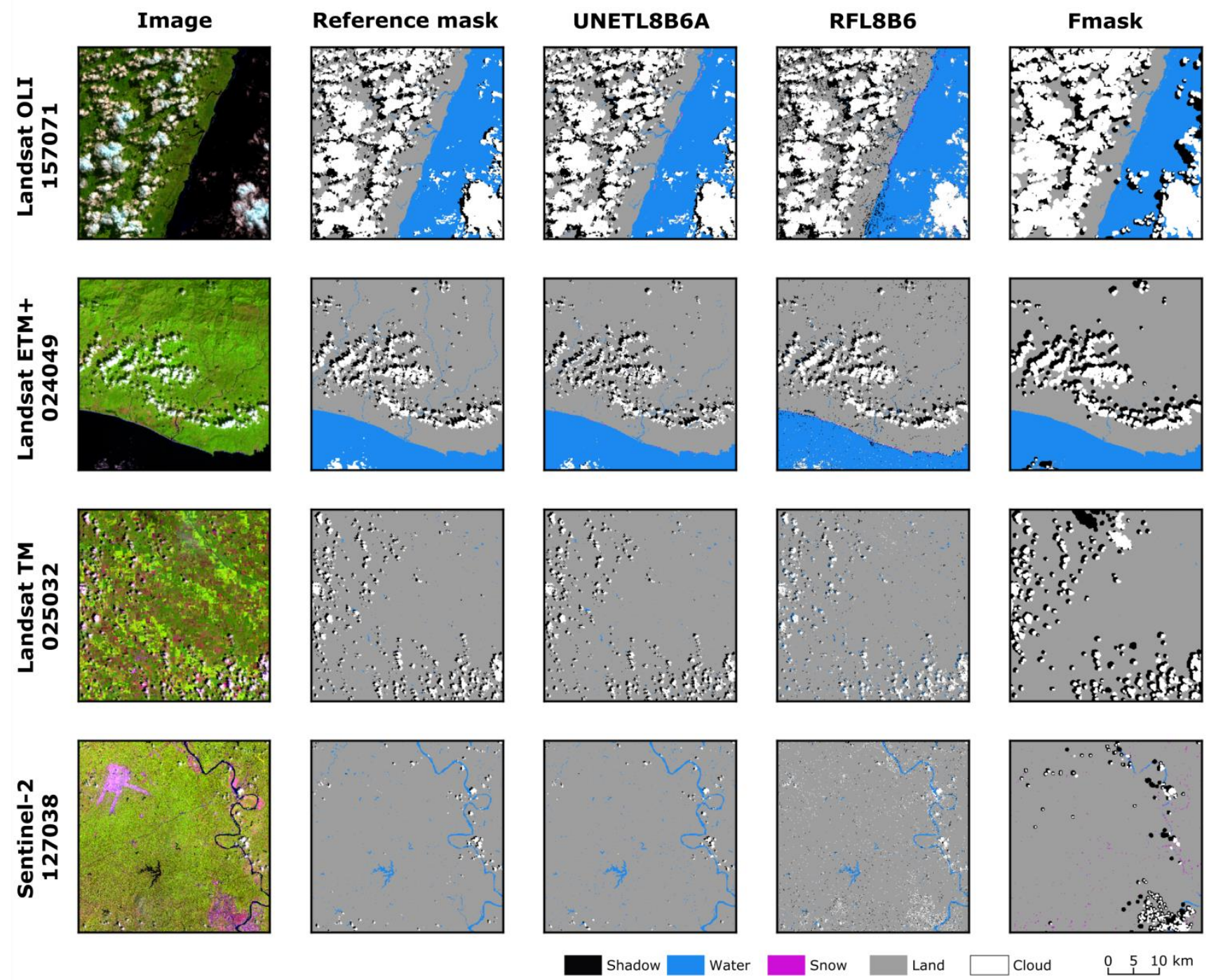

Figure 8: Selected results for the best performing U-Net and Random Forest models and Fmask on Landsat OLI 330 for different sensors and locations. 


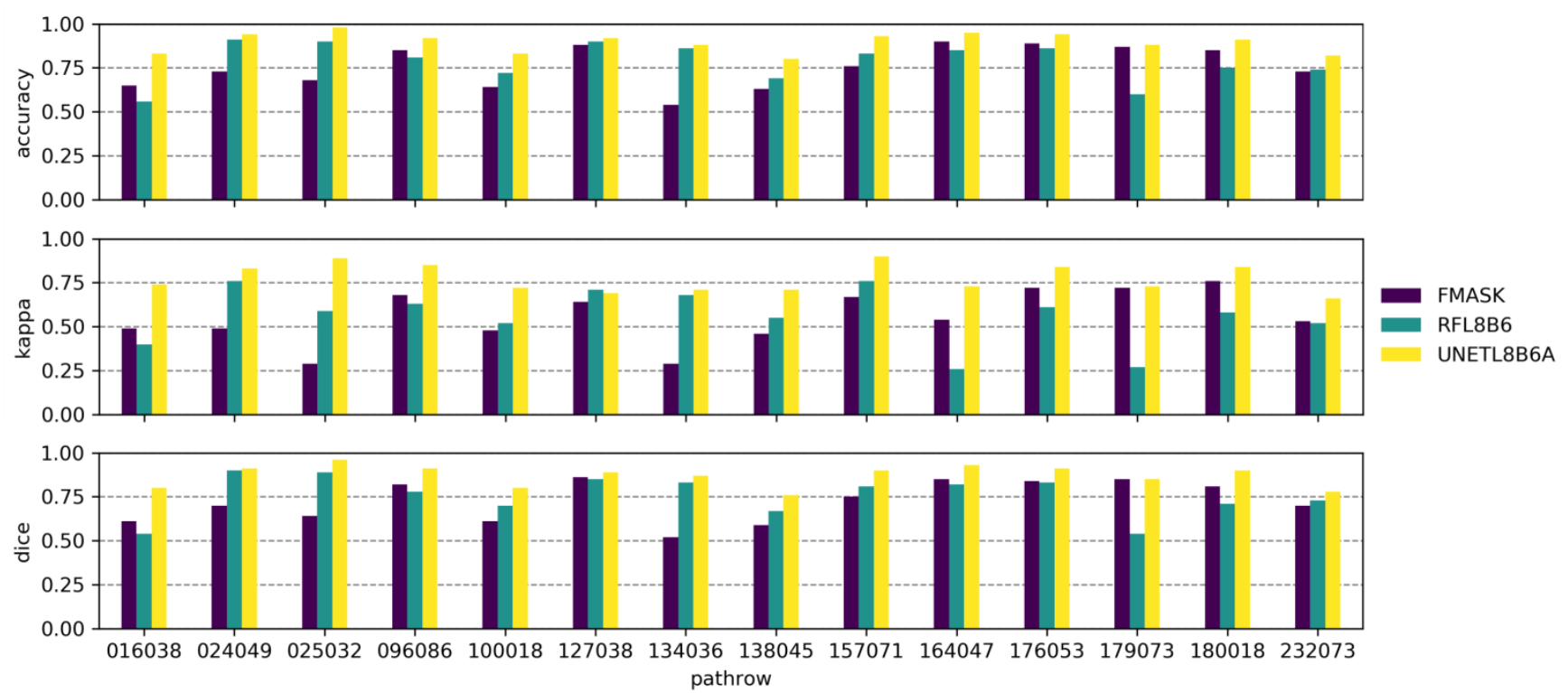

Figure 9: Summary of the results for the best performing U-Net and Random Forest models and Fmask, grouped by location.

\section{Discussion}

In this study, we used a freely available global training dataset based on Landsat OLI and developed a multisensor reference dataset that we used for testing our models. Our reference dataset has been sampled globally under consideration of terrestrial biomes to account for a large variety of climatic, atmospheric and land-cover conditions. Training, validation and test masks are derived by manual interpretation and digitization of the respective satellite images. The results presented in this study are therefore to be regarded as relative to the performance of a human analyst and not to an absolute ground-truth. Especially clouds and shadows have fuzzy outlines and their interpretation and delineation are at least partially subjective. Moreover, cross-sensor comparison may be biased by a temporal shift between acquisition dates at single test sample locations. This is particularly prominent when comparing Landsat TM and ETM+ with Landsat OLI and Sentinel-2, due to the non-overlapping lifetime of the satellites. We tried to minimize this temporal bias by choosing images that were acquired during the same season for each sample location.

We trained a U-Net CNN using different training strategies and spectral band combinations derived from the SPARCS dataset. The results were compared to a Random Forest classifier and the widely used Fmask method. We could show that even U-Net models that use only four spectral bands (Red, Green, Blue and NIR) can produce competitive prediction results. This becomes particularly relevant when sensors with limited spectral resolution, such as RapidEye or Planet Scope, are targeted by an application. Adding SWIR bands to the input feature space could improve the results by 0.04 for Kappa over all sensors and locations compared to using visible and NIR bands alone (Table 2). Atmospheric aerosols have a stronger effect on shorter wavelengths (mainly in the visible spectrum), whereas the SWIR wavelengths show improved atmospheric transparency and 
can aid in the distinction between clouds, snow and ice. Augmentation proved to be important for the network to learn invariance to changes in the target domain, which is strongly affected by atmospheric conditions, seasonality, land-use/land-cover and other scene and image properties such as sun elevation or radiometric resolution. However, this statement only holds for the six band training data where an improvement of 0.05 for Kappa compared to the non-augmented training data could be observed over all sensors (Table 2). Augmenting the four band training data resulted in a reduction of the performance by 0.02 for Kappa over all sensors. Interestingly, this reduction seems to be driven by the performance on the Landsat sensors, as an increase for Kappa can be observed for Sentinel-2. It seems that the simple contrast/brightness augmentation could not sufficiently account for the variations in the visible and NIR target domain and potentially even introduced noise, which bias the network and lead to a higher risk of overfitting (Figure 5) and reduced generalization ability of the model. More research into other transformations or more sophisticated augmentation techniques (e.g., generating synthetic training samples via Generative Adversarial Networks) would be needed to investigate their potential to account for more complex variations and to improve the performance given a reduced four band feature space. More experiments should be conducted to understand the causes for cross-sensor differences.

Compared to our method, Fmask also utilizes the Thermal Infrared (TIR) bands where available. The results of Fmask seem to be strongly dependent on the availability of TIR bands as can be seen in the performance decrease from Landsat (with thermal bands) to Sentinel-2 (no thermal bands) by 0.15-0.27 for Kappa (Table 2). We specifically decided against using the TIR bands in our study in favour of a higher degree of transferability across sensors. For sensor-specific applications, however, including TIR bands into the input feature space of the network may have a positive effect on the accuracy.

Fmask is a transparent rule-set that can be closely related to physical and geometrical remote sensing principals. Compared to such rule-based and expert-driven approaches, CNNs are less transparent and further fundamental research is needed to better understand their internal reasoning. Random Forest is a well-understood machinelearning method with a relatively high degree of transparency. Nevertheless, in the given experimental setup Random Forest produced consistently lower accuracies than the CNN models. Even the best performing Random Forest model seems to have significant problems to distinguish between "shadow" and "water" classes. Shadow prevents the spectral characteristics of the underlying surface from being fully represented, which may cause an overlap of the spectral signatures of shadow and water pixels. Classifiers that only consider the spectral characteristics of single pixels in their input feature space, like the Random Forest classifier used in this study, may hence inevitably have problems to distinguish these classes. The CNN models seem to largely overcome this problem by their non-linear function mapping capability, and by the convolutional features that are being learned directly from the data under consideration of neighbourhood information at various scales. In case of Random Forest, the use of additional hand-crafted features (e.g., first and second order statistics or high-level features like bag of visual words) computed over a sliding-window or as part of an object-based image analysis could improve the results, but would also involve longer computation time during inference. 
In the tested setup, Random Forest has the shortest inference time when running on CPU, followed by U-Net and Fmask. We showed that a modern GPU can highly accelerate inference times of the U-Net models that even outperform Random Forest models. The comparably long inference times of Fmask are likely related to the fact that the method needs to compute several additional layers (view angles of the satellite sensor, illuminating angle and estimates of cloud altitude from thermal information) before the actual rule-set can be applied. It should be noted that we use Fmask as a Python package, which may also influence computation time compared to using for example CFmask directly. Recently, Fmask results are provided as part of the quality assessment band of Landsat Collection-1 data. However, we have decided to run Fmask ourselves in order to consistently compare processing times across different sensors and methods.

Hughes and Hayes (2014) (Hughes and Hayes, 2014), who developed the SPARCS dataset, achieve highly accurate results by using a traditional neural network and rule-based spatial post-processing. They train and test their model on independent subsets of the same reference data and specifically target Landsat OLI in their study. Compared to their study, we do not apply any post-processing steps and focus on multi-sensor generalization ability. Accordingly, we test our results against an independently acquired multi-sensor dataset with potentially more complex variations in the target domain. Similar to our study, Zhaoxiang et al. (2018) (Zhaoxiang et al., 2018) use a lightweight U-Net, but train and test it exclusively on a modified version of the SPARCS dataset. Their focus lies on on-board cloud detection and accordingly they consider a binary classification task, with minimal feature space (Red, Green, Blue and NIR bands) and investigate the influence of image compression on the results. Isikdogan, Bovik and Passalacqua (2017) (Isikdogan et al., 2017) use a Fully Convolutional Network to segment an image into the same classes as used in our study with their main objective being surface water mapping. Similarly to the aforementioned studies, they train and test their models on independent subsets of the same dataset (Landsat ETM+ with corresponding label masks from the GLCF inland water dataset (Feng et al., 2016)), but do not specifically target transferability between sensors.

\section{Conclusions}

413 In this study, we introduced a rapid and simple to use method to segment multi-spectral satellite images into five 414 major classes including "cloud" and "cloud shadow", which are particularly relevant for any application that 415 uses optical data. The proposed U-Net CNN has been trained on different variations of the freely available 416 SPARCS dataset (based on Landsat OLI) (USGS, 2016). The best performing model uses six spectral bands 417 (Red, Green, Blue, NIR, SWIR1 and SWIR2) and has been trained on a contrast and brightness augmented 418 version of the training data. We showed that it is possible for a single model to generalize between different 419 satellite sensors and locations without the need for atmospheric correction, relative normalization, re-training, or 420 other domain adaptation techniques. Our method consistently outperformed the widely used Fmask and Random 421 Forest methods in terms of accuracy, Cohen's Kappa coefficient, Dice coefficients, and inference time on a 422 globally sampled test dataset derived from Landsat TM, ETM+, OLI and Sentinel-2 data. Future research efforts 
will focus on the development and evaluation of a new training dataset that incorporates data from different sensors and more locations world-wide with the aim to further improve the accuracy, while decreasing performance variations across sensors and locations. Furthermore, it is planned to test the method on additional medium to high resolution sensors (e.g., RapidEye, ALOS-AVNIR2, Planet Scope), and to adapt the method to very high resolution satellite and aerial imagery (e.g., WorldView, Pleiades), which impose particular challenges related to their sub-meter spatial resolution and the limited spectral resolution. The findings of this study will be applied within a flood monitoring system that uses imagery from multiple SAR and optical satellite sensors to produce timely flood maps for situational awareness in emergency situations (Martinis et al., 2013; Twele et al., 2016). Compared to other available solutions, the proposed method allows us to rapidly and accurately segment cloud and cloud shadow pixels in a large range of multi-spectral satellite image types without relying on ancillary datasets, multi-temporal analysis, or manual adjustments of rule-sets and parameters. Being able to quickly and accurately identify cloud and cloud shadow pixels is fundamental for unbiased down-stream analysis, particularly in emergency applications where time is critical and all available data sources need to be utilized to provide timely information products about an evolving disaster situation.

\section{Acknowledgements}

This work was supported by the German Federal Ministry of Education and Research (BMBF) as part of the RIESGOS project (Grant No. 03G0876). The authors thank the anonymous reviewers for their constructive comments and suggestions that helped to improve the manuscript.

\section{References}

Bai, T., Li, D., Sun, K., Chen, Y., Li, W., 2016. Cloud Detection for High-Resolution Satellite Imagery Using Machine Learning and Multi-Feature Fusion. Remote Sens. 8, 715. https://doi.org/10.3390/rs8090715 Banskota, A., Kayastha, N., Falkowski, M.J., Wulder, M.A., Froese, R.E., White, J.C., 2014. Forest Monitoring Using Landsat Time Series Data: A Review. Can. J. Remote Sens. 40, 362-384. https://doi.org/10.1080/07038992.2014.987376

Breiman, L., 2001. Random Forests. Mach. Learn. 45, 5-32. https://doi.org/10.1023/A:1010933404324

Chen, Y., Fan, R., Bilal, M., Yang, X., Wang, J., Li, W., 2018. Multilevel Cloud Detection for High-Resolution Remote Sensing Imagery Using Multiple Convolutional Neural Networks. ISPRS Int. J. Geo-Inf. 7, 181. https://doi.org/10.3390/ijgi7050181

Dosovitskiy, A., Springenberg, J.T., Brox, T., 2014. Unsupervised feature learning by augmenting single images. ArXiv:1312.5242 7.

Feng, M., Sexton, J.O., Channan, S., Townshend, J.R., 2016. A global, high-resolution (30-m) inland water body dataset for 2000: first results of a topographic-spectral classification algorithm. Int. J. Digit. Earth 9, 113-133. https://doi.org/10.1080/17538947.2015.1026420 
Foga, S., Scaramuzza, P.L., Guo, S., Zhu, Z., Dilley, R.D., Beckmann, T., Schmidt, G.L., Dwyer, J.L., Joseph Hughes, M., Laue, B., 2017. Cloud detection algorithm comparison and validation for operational Landsat data products. Remote Sens. Environ. 194, 379-390. https://doi.org/10.1016/j.rse.2017.03.026

Goodman, A.H., Henderson-Sellers, A., 1988. Cloud detection and analysis: A review of recent progress. Atmospheric Res. 21, 203-228. https://doi.org/10.1016/0169-8095(88)90027-0

Hagolle, O., Huc, M., Pascual, D.V., Dedieu, G., 2010. A multi-temporal method for cloud detection, applied to FORMOSAT-2, VEN $\mu$ S, LANDSAT and SENTINEL-2 images. Remote Sens. Environ. 114, 17471755. https://doi.org/10.1016/j.rse.2010.03.002

Hollstein, A., Segl, K., Guanter, L., Brell, M., Enesco, M., 2016. Ready-to-Use Methods for the Detection of Clouds, Cirrus, Snow, Shadow, Water and Clear Sky Pixels in Sentinel-2 MSI Images. Remote Sens. 8, 666. https://doi.org/10.3390/rs8080666

Hughes, M., Hayes, D., 2014. Automated Detection of Cloud and Cloud Shadow in Single-Date Landsat Imagery Using Neural Networks and Spatial Post-Processing. Remote Sens. 6, 4907-4926. https://doi.org/10.3390/rs6064907

Hutchison, K.D., Mahoney, R.L., Vermote, E.F., Kopp, T.J., Jackson, J.M., Sei, A., Iisager, B.D., 2009. A Geometry-Based Approach to Identifying Cloud Shadows in the VIIRS Cloud Mask Algorithm for NPOESS. J. Atmospheric Ocean. Technol. 26, 1388-1397. https://doi.org/10.1175/2009JTECHA1198.1 Isikdogan, F., Bovik, A.C., Passalacqua, P., 2017. Surface Water Mapping by Deep Learning. IEEE J. Sel. Top. Appl. Earth Obs. Remote Sens. 10, 4909-4918. https://doi.org/10.1109/JSTARS.2017.2735443

Keras [WWW Document], 2018. URL https://keras.io (accessed 6.14.18).

Kingma, D.P., Lei, J., 2015. Adam: A Method for Stochastic Optimization. ArXiv:1412.6980v9 15.

Lee, K.-Y., Lin, C.-H., 2016. Cloud detectino of optical satellite images using Support Vector Machine. ISPRS Int. Arch. Photogramm. Remote Sens. Spat. Inf. Sci. XLI-B7, 289-293. https://doi.org/10.5194/isprsarchives-XLI-B7-289-2016

Li, Z., Shen, H., Li, H., Xia, G., Gamba, P., Zhang, L., 2017. Multi-feature combined cloud and cloud shadow detection in GaoFen-1 wide field of view imagery. Remote Sens. Environ. 191, 342-358. https://doi.org/10.1016/j.rse.2017.01.026

Long, J., Shelhamer, E., Darrell, T., 2015. Fully Convolutional Networks for Semantic Segmentation. Presented at the IEEE Conference on Computer Vision and Pattern Recognition (CVPR), IEEE, Boston, USA, p. 10. https://doi.org/10.1109/CVPR.2015.7298965

Luo, Y., Trishchenko, A., Khlopenkov, K., 2008. Developing clear-sky, cloud and cloud shadow mask for producing clear-sky composites at 250-meter spatial resolution for the seven MODIS land bands over Canada and North America. Remote Sens. Environ. 112, 4167-4185. https://doi.org/10.1016/j.rse.2008.06.010

Lyapustin, A., Wang, Y., Frey, R., 2008. An automatic cloud mask algorithm based on time series of MODIS 
measurements. J. Geophys. Res. 113. https://doi.org/10.1029/2007JD009641

Main-Knorn, M., Pflug, B., Louis, J., Debaecker, V., Müller-Wilm, U., Gascon, F., 2017. Sen2Cor for Sentinel2, in: Bruzzone, L., Bovolo, F., Benediktsson, J.A. (Eds.), SPIE Image and Signal Processing for Remote Sensing XXIII. Warsaw, Poland, p. 3. https://doi.org/10.1117/12.2278218

Martinis, S., Twele, A., Strobl, C., Kersten, J., Stein, E., 2013. A Multi-Scale Flood Monitoring System Based on Fully Automatic MODIS and TerraSAR-X Processing Chains. Remote Sens. 5, 5598-5619. https://doi.org/10.3390/rs5115598

Mateo-Garcia, G., Gomez-Chova, L., Camps-Valls, G., 2017. Convolutional neural networks for multispectral image cloud masking, in: 2017 IEEE International Geoscience and Remote Sensing Symposium (IGARSS). Presented at the 2017 IEEE International Geoscience and Remote Sensing Symposium (IGARSS), IEEE, Fort Worth, TX, pp. 2255-2258. https://doi.org/10.1109/IGARSS.2017.8127438

Oishi, Y., Ishida, H., Nakamura, R., 2018. A new Landsat 8 cloud discrimination algorithm using thresholding tests. Int. J. Remote Sens. 1-21. https://doi.org/10.1080/01431161.2018.1506183

Olson, D.M., Dinerstein, E., Wikramanayake, E.D., Burgess, N.D., Powell, G.V.N., Underwood, E.C., D’amico, J.A., Itoua, I., Strand, H.E., Morrison, J.C., Loucks, C.J., Allnutt, T.F., Ricketts, T.H., Kura, Y., Lamoreux, J.F., Wettengel, W.W., Hedao, P., Kassem, K.R., 2001. Terrestrial Ecoregions of the World: A New Map of Life on Earth. BioScience 51, 933. https://doi.org/10.1641/00063568(2001)051[0933:TEOTWA]2.0.CO;2

Ozkan, S., Efendioglu, M., Demirpolat, C., 2018. Cloud detection from RGB rcolor remote sensing images with deep pyramid networks. ArXiv:1801.08706v1 4.

Pankiewicz, G.S., 2007. Pattern recognition techniques for the identification of cloud and cloud systems. Meteorol. Appl. 2, 257-271. https://doi.org/10.1002/met.5060020309

Pekel, J.-F., Cottam, A., Gorelick, N., Belward, A.S., 2016. High-resolution mapping of global surface water and its long-term changes. Nature 540, 418-422. https://doi.org/10.1038/nature20584

Python Fmask [WWW Document], 2018. URL http://pythonfmask.org/en/latest/ (accessed 6.29.18).

Ronneberger, O., Fischer, P., Brox, T., 2015. U-Net: Convolutional Networks for Biomedical Image Segmentation, in: Navab, N., Hornegger, J., Wells, W.M., Frangi, A.F. (Eds.), Medical Image Computing and Computer-Assisted Intervention - MICCAI 2015. Springer International Publishing, Cham, pp. 234-241. https://doi.org/10.1007/978-3-319-24574-4_28

Roy, D.P., Ju, J., Kline, K., Scaramuzza, P.L., Kovalskyy, V., Hansen, M., Loveland, T.R., Vermote, E., Zhang, C., 2010. Web-enabled Landsat Data (WELD): Landsat ETM+ composited mosaics of the conterminous United States. Remote Sens. Environ. 114, 35-49. https://doi.org/10.1016/j.rse.2009.08.011

Scikit-learn [WWW Document], 2018. URL http://scikit-learn.org (accessed 5.8.18).

Sholar, J.M., 2017. Lightweight Deconvolutional Neural Networks for Efficient Cloud Identification in Satellite Images (No. 561), CS231N. Stanford University, Stanford, USA. 
Simonyan, K., Zisserman, A., 2014. Very deep convolutional networks for large-scale image recognition. ArXiv Prepr. ArXiv14091556.

Smith, L.N., 2017. Cyclical Learning Rates for Training Neural Networks, in: 2017 IEEE Winter Conference on Applications of Computer Vision (WACV). Presented at the 2017 IEEE Winter Conference on Applications of Computer Vision (WACV), IEEE, Santa Rosa, CA, USA, pp. 464-472. https://doi.org/10.1109/WACV.2017.58

Twele, A., Cao, W., Plank, S., Martinis, S., 2016. Sentinel-1-based flood mapping: a fully automated processing chain. Int. J. Remote Sens. 37, 2990-3004. https://doi.org/10.1080/01431161.2016.1192304

USGS, L., 2018. Landsat Band Designations [WWW Document]. URL https://landsat.usgs.gov/what-are-banddesignations-landsat-satellites (accessed 1.2.18).

USGS, L., 2013. Using the USGS Landsat Level-1 Data Product [WWW Document]. URL https://landsat.usgs.gov/using-usgs-landsat-8-product

USGS, S., 2016. L8 SPARCS Cloud Validation Masks. U.S. Geological Survey data release [WWW Document]. URL https://landsat.usgs.gov/sparcs (accessed 7.4.18).

Voigt, S., Giulio-Tonolo, F., Lyons, J., Kučera, J., Jones, B., Schneiderhan, T., Platzeck, G., Kaku, K., Hazarika, M.K., Czaran, L., Li, S., Pedersen, W., James, G.K., Proy, C., Muthike, D.M., Bequignon, J., GuhaSapir, D., 2016. Global trends in satellite-based emergency mapping. Science 353, 247-252. https://doi.org/10.1126/science.aad8728

Wieland, M., 2016. Large-area settlement pattern recognition from Landsat-8 data. ISPRS J. Photogramm. Remote Sens. 119, 294-308.

Zhan, Y., Wang, J., Shi, J., Cheng, G., Yao, L., Sun, W., 2017. Distinguishing Cloud and Snow in Satellite Images via Deep Convolutional Network. IEEE Geosci. Remote Sens. Lett. 14, 1785-1789. https://doi.org/10.1109/LGRS.2017.2735801

Zhaoxiang, Z., Iwasaki, A., Guodong, X., Jianing, S., 2018. Small Satellite Cloud Detection Based On Deep Learning and Image Compression. Preprints. https://doi.org/10.20944/preprints201802.0103.v1

Zhu, X.X., Tuia, D., Mou, L., Xia, G.-S., Zhang, L., Fraundorfer, F., 2017. Deep Learning in Remote Sensing: A Review. IEEE Geosci. Remote Sens. Mag. 60.

Zhu, Z., Wang, S., Woodcock, C.E., 2015. Improvement and expansion of the Fmask algorithm: cloud, cloud shadow, and snow detection for Landsats 4-7, 8, and Sentinel 2 images. Remote Sens. Environ. 159, 269-277. https://doi.org/10.1016/j.rse.2014.12.014

Zhu, Z., Woodcock, C.E., 2012. Object-based cloud and cloud shadow detection in Landsat imagery. Remote Sens. Environ. 118, 83-94. https://doi.org/10.1016/j.rse.2011.10.028

Zi, Y., Xie, F., Jiang, Z., 2018. A Cloud Detection Method for Landsat 8 Images Based on PCANet. Remote Sens. 10, 877. https://doi.org/10.3390/rs10060877 


\section{$561 \quad$ List of figure captions}

562 Figure 1: Worldwide Reference System 2 (WRS2) path/row locations of training, validation and testing data. 563 Training and validation data are derived from the SPARCS dataset (Landsat OLI) (USGS, 2016). Stratified 564 random sampling has been applied on the basis of a global biomes map (Olson et al., 2001) to choose WRS2 565 path/row locations for independent testing data from multiple sensors (Landsat TM, ETM+, OLI and Sentinel-2). 566 Testing data samples are labelled with their unique identifier given by the respective path/row combination.

$567 \quad$ Figure 2: Comparison of spectral bands and spatial resolution across different satellite sensors superimposed 568 on atmospheric transmission in grey. Modified after (USGS, 2018).

569 Figure 3: Overview of the performance evaluation framework.

$570 \quad$ Figure 4: Architecture of the U-Net used in this study.

571 Figure 5: Visualization of the training history for U-Net models. A description of the different training setups 572 can be found in Table 1 (e.g., UNETL8B4A refers to a U-Net model that has been trained on four Landsat OLI 573 bands with contrast/brightness augmentation).

574 Figure 6: Summary of the results for each tested satellite sensor, grouped by model.

575 Figure 7: Error matrices for the best performing U-Net and Random Forest models and Fmask over the 896 test 576 tiles for Landsat TM, ETM+, OLI and Sentinel-2.

577 Figure 8: Selected results for the best performing U-Net and Random Forest models and Fmask on Landsat OLI 578 for different sensors and locations.

579 Figure 9: Summary of the results for the best performing U-Net and Random Forest models and Fmask, grouped 580 by location. 